\title{
Patient outcomes up to 15 years after stroke: survival, disability, quality of life, cognition and mental health
}

\author{
Siobhan L Crichton, ${ }^{1,2}$ Benjamin D Bray, ${ }^{3}$ Christopher McKevitt, ${ }^{1,2}$ \\ Anthony G Rudd, ${ }^{1,2}$ Charles D A Wolfe ${ }^{1,2,4}$
}

- Additional material is published online only. To view please visit the journal online (http://dx.doi.org/10.1136/ innp-2016-313361)

${ }^{1}$ Division of Health and Social Care Research, King's College London, London, UK ${ }^{2}$ NIHR Biomedical Research Centre, Guy's \& St. Thomas' NHS Foundation Trust and King's College London, London, UK

${ }^{3}$ Farr Institute of Health Informatics, University College London, London, UK

${ }^{4}$ National Institute of Health Research Collaboration for Leadership in Applied Health Research and (are (CLAHRC) South London, London, UK

\section{Correspondence to} Siobhan Crichton, Division of Health and Social Care Research, Faculty of Life Sciences \& Medicine, King's College London, 5th Floor Addison House, [1] Guy's Campus, London SE1 1UL, UK; Siobhan.crichton@kcl.ac.uk

BDB and SLC are joint first authors.

Received 17 February 2016 Revised 23 June 2016 Accepted 29 June 2016 Published Online First 22 July 2016

\section{ABSTRACT}

Background The global epidemiological shift of disease burden towards long-term conditions means understanding long-term outcomes of cardiovascular disease is increasingly important. More people are surviving stroke to experience its long-term consequences, but outcomes in people living more $>10$ years after stroke have not been described in detail. Methods Data were collected for the population-based South London Stroke Register, with participants followed up annually until death. Outcomes were survival, disability, activity, cognitive impairment, quality of life, depression and anxiety.

Findings of 2625 people having first-ever stroke, 262 $(21 \%)$ survived to 15 years. By 15 years, $61 \%(95 \% \mathrm{Cl}$ $55 \%$ to $67 \%$ ) of the survivors were male, with a median age of stroke onset of 58 years (IQR 48-66). 87\% of the 15 -year survivors were living at home and $33.8 \%$ (26.2\% to $42.4 \%$ ) had mild disability, $14.3 \%$ (9.2\% to $21.4 \%)$ moderate disability and $15.0 \%(9.9 \%$ to $22.3 \%)$ severe disability. The prevalence of disability increased with time but 1 in 10 of the 15-year survivors had lived with moderate-severe disability since their stroke. At 15 years, the prevalence of cognitive impairment was $30.0 \%$ (19.5\% to $43.1 \%)$, depression $39.1 \%(30.9 \%$ to $47.9 \%)$ and anxiety $34.9 \%$ (27.0\% to $43.8 \%$ ), and survivors reported greater loss of physical than mental quality of life.

Conclusions One in five people live at least 15 years after a stroke and poor functional, cognitive and psychological outcomes affect a substantial proportion of these long-term survivors. As the global population of individuals with cardiovascular long-term conditions grows, research and health services will need to increasingly focus on preventing and managing the long-term consequences of stroke.

\section{INTRODUCTION}

Stroke affects an estimated 17 million people each year globally, ${ }^{1}$ a number projected to increase as a result of demographic changes and a greater number of people living to older ages where the risk of stroke is greatest. The Global Burden of Disease study has estimated that despite falling age-standardised rates of incident stroke, the number of strokes occurring worldwide increased by $68 \%$ and the number of stroke survivors by $84 \%$ between 1990 and 2010. ${ }^{1}$ Owing to improvements in acute stroke care such as organised inpatient stroke unit-based care, ${ }^{2}$ a greater proportion of patients are surviving the acute stroke episode. ${ }^{3}$ For many survivors and their families, this results in living a life affected by the long-term consequences of stroke, such as physical disability, cognitive impairment, ${ }^{4}$ fatigue and psychological problems such as depression and anxiety. ${ }^{5}$ Stroke is therefore shifting away from being a major killer to becoming a long-term (chronic) condition with multiple impacts on individuals, health systems and society. ${ }^{16}$

Our current knowledge of the long-term consequences of stroke is limited by the short follow-up of most previous studies of stroke outcomes. There are few studies of outcomes 5-10 years after stroke, ${ }^{7-9}$ and previous studies of outcomes more than 10 years after stroke have included only small numbers of patients (60 in total from populationbased registers) and described only a small number of outcomes ${ }^{7}{ }^{10-15}$ (summarised in online supplementary table SA). As a result, there have been very little data available to forecast what will be the consequences of the epidemiological shift towards stroke being a long-term condition. Outcomes data from other cardiovascular conditions such as myocardial infarction are also lacking for longer periods of follow-up and limited largely to survival rather than other health outcomes; ${ }^{16}{ }^{17}$ describing the long-term outcomes of stroke therefore contributes to a broader understanding of the population impact of cardiovascular long-term conditions.

We therefore aimed to estimate the long-term outcomes of stroke across multiple domains of health (survival, functional, cognitive, quality of life and mental health) up to 15 years after stroke, using data from a population-based register designed to identify all people with first-ever stroke in a geographically defined area of London.

\section{METHODS}

Study population

The South London Stroke Register (SLSR) is a prospective population-based stroke register set up in January 1995, recording all first-ever strokes in patients of all ages for an inner area of South London based on 22 electoral wards in Lambeth and Southwark.

At the time of the 2001 census, the SLSR source population consisted of 271817 individuals (63\% white, 24\% black and 9\% other ethnic groups). The population rose to 357308 (56\% white, 25\% black and $18 \%$ other ethnic groups) by the time of 
the 2011 census and is more ethnically diverse than the UK as a whole (86\% white).

\section{Case ascertainment}

Methods used in data collection have been previously described $^{18}$ and are summarised below. Standardised criteria were applied to ensure completeness of case ascertainment, including multiple overlapping sources of notification. ${ }^{18}$ Stroke was defined according to the WHO criteria, ${ }^{18}$ and all subarachnoid haemorrhages (International Classification of Disease 10th Edition (ICD-10) code I60), intracerebral haemorrhages (I61), cerebral infarctions (I63) and unspecified strokes (I64) were included. Patients admitted to hospitals serving the study area were identified by regular reviews of acute wards admitting patients with stroke, checks of brain imaging and monthly reviews of bereavement officer and bed manager records. Death certificates were checked regularly. Completeness of case ascertainment has been estimated at $88 \%$ by a multinomial-logit capture-recapture model using the methods described in detail elsewhere. ${ }^{19}$ Registration of first stroke recurrence was performed in the same way as the initial stroke. ${ }^{20}$

\section{Data collection}

Trained study nurses and field workers collected the data and a study doctor verified the diagnosis of stroke. Participants were examined within 48 hour of referral to SLSR where possible. They were assessed at 3 months and annually after stroke. Follow-up data were collected using postal questionnaires or by fieldworks in face-to-face interviews with patients and/or carers. Participants who had left the SLSR area were followed up where possible using telephone interviews or postal questionnaires. The validated instruments used to measures outcomes included activities of daily living (ADL) using the Barthel Index (BI), ${ }^{21}$ extended ADL using the Frenchay Activities Index (FAI), ${ }^{22}$ cognitive impairment using the Abbreviated Mental Test (AMT), ${ }^{23}$ anxiety and depression using the Hospital Anxiety and Depression Scale (HADS) ${ }^{24}$ and health-related quality of life (HRQOL) using the Short Form 12 (SF-12). ${ }^{22}$

Cut-off points for defining outcomes were defined a priori. A score on the BI of 0-9 was used to identify patients with severe (BI <9) disability, 10-14 for moderate, 15-19 for mild and 20 to identify those who were independent. ${ }^{25}$ The FAI was used to categorise participants with a score $<16$ categorised as 'inactive', $16-30$ as moderately active and $31+$ as active. $^{26}$

Participants were defined as cognitively impaired if they had an AMT score $<8 .^{27}$ The HADS consists of two subscales and each subscale was used to identify cases of anxiety and depression (score $>7) .^{28}$

HRQOL was measured using the SF-36 in follow-ups to 1 March 1999, when it was replaced with the SF-12. The 12 items of the SF-12 have been adopted from the SF-36 verbatim, and summary scores are replicable and reproducible. ${ }^{29}$ Therefore, items from the SF-36 questionnaires in earlier follow-ups were used to derive SF-12 summary scores across all time points. The SF-12 is summarised using two domains which represent physical and mental health. ${ }^{22}$ The summary scores range from 0 to 100 and are based on population norms with a mean of 50 and an SD of 10 .

Data included were of patients with a first-ever stroke between 1 January 1995 and 31 December 2003. Information on long-term outcomes of surviving participants was extracted from the annual follow-up to 15 years after stroke. Survival time was defined as time between stroke onset and death, using date of death extracted from the national statutory register of deaths. For participants still alive on 31 December 2014, survival time was censored and defined as time between stroke onset and the end of 2014. Survival free of recurrence was defined as time between initial stroke onset and time of second stroke. Patients were censored on 31 December 2014 or at the time of death, whichever was soonest, meaning not all patients had a full 15 years of follow-up.

\section{Statistical analysis}

Kaplan-Meier estimates were used to model survival and to measure the cumulative survival and cumulative survival free of recurrence with $95 \%$ confidence intervals (CIs) up to 15 years after stroke. The characteristics of all participants and those who were alive at 10 and 15 years after stroke were summarised as frequency (percentage) or median (IQR). The lost to follow-up rate at each time point was calculated as the percentage of participants who were alive but missed the interview. Binomial CIs were calculated for point prevalence estimates. The characteristics of participants who were alive and followed up at each time point were compared to those who were lost to follow-up using $\chi^{2}$ and Mann-Whitney U tests. Long-term outcomes were summarised as the proportions in each of the predefined BI, FAI, AMT and HADS categories. A poor outcome was defined as moderate-severe disability, inactivity, cognitive impairment, anxiety or depression and the number of poor outcomes reported by participants at each time point calculated. All data available at each time point were included and participants who were lost to follow-up at one point remained eligible for inclusion in later follow-ups.

Analysis was conducted using Stata V.13MP and R 2.15.1

\section{Ethics}

All patients or their relatives gave written informed consent to participate in the study, and over the study period very few patients have declined to be registered. The design of the study was approved by the ethics committees of Guy's and St Thomas' National Health Service Foundation Trust, King's College Hospital Foundation Trust, St George's University Hospital, National Hospital for Nervous Diseases, and Westminster Hospital.

\section{RESULTS}

Between 1 January 1995 and 31 December 2003, 2626 firstever strokes were recorded. The follow-up rates among survivors at 10 and 15 years after stroke were $56.7 \%$ and $50.8 \%$, respectively. A full breakdown of the number of participants eligible for each follow-up and the numbers completing follow-up is available in online supplementary figure S1. At 10 years, those who were lost to follow-up were on average 3.4 years younger than those who were followed up $(p=0.001)$. There were no significant differences in the characteristics of participants who were lost to follow-up in terms of sex $(p=0.789$ and 0.707$)$, ethnicity $(p=0.273$ and $p=0.273)$ or stroke type $(p=0.588$ and $=0.243$ ) at either time point or in age at 15 years $(\mathrm{p}=0.128)$.

Participants were followed for a total of 14184 person years (mean $=5.4$ years in all participants and 13.5 years in those still alive at 10 years after stroke). By 10 years after stroke, 1903 participants had died and 318 recurrent strokes were recorded. The corresponding figures at 15 years were 2066 deaths and 333 recurrences and 298 participants had not yet reached the 15 -year follow-up point (see online supplementary figure S1 for numbers of deaths and recurrences other time points). Cumulative survival was $28.9 \%$ (95\% CI $27.0 \%$ to $30.1 \%$ ) at 10 years and $20.6 \%(19.0 \%$ to $22.4 \%$ ) at 15 years (figure 1 ), 


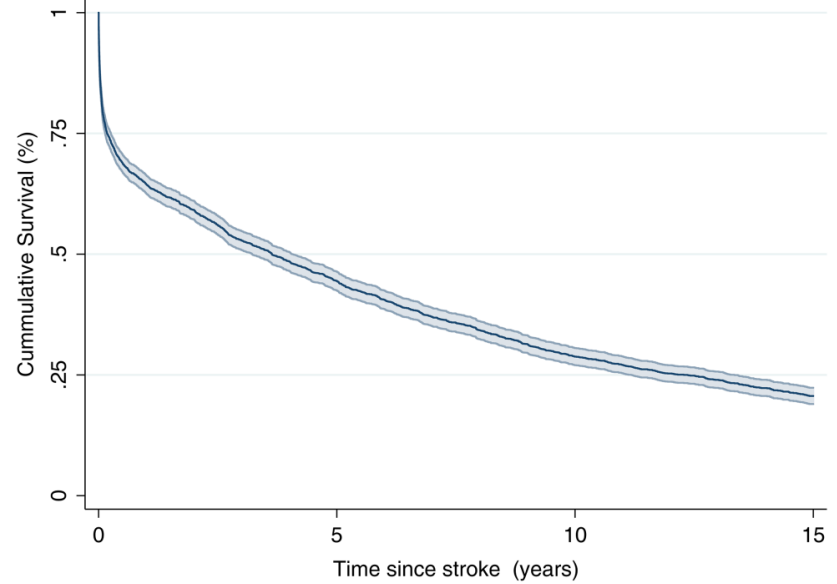

Figure 1 Kaplan-Meier survival estimates with $95 \% \mathrm{Cl}$.

and at 10 years $65.3 \%(61.4 \%$ to $68.9 \%)$ of survivors were recurrence free $(54.4 \%(47.4 \%$ to $60.9 \%)$ at 15 years; tables 1 and 2).

The demographic characteristics of all participants at the time of stroke, and of those who survived 10 and 15 years are summarised in table 1 . The median age at stroke onset was 73 years (IQR 63-81) and 50.5\% (95\% CI 48.6\% to 52.4\%) occurred in females. The majority of participants were of white ethnicity $(74.5 \%, 72.8 \%$ to $76.1 \%)$. Fewer females than males survived to 10 and 15 years, by which time females constituted $41.2 \%$ (37.7\% to $44.9 \%)$ and $39.3 \%$ (33.5\% to $45.4 \%)$ of survivors, respectively. Participants of Black ethnicity were also more likely to survive and represented almost one-third of long-term survivors $(29.6 \%, 26.4 \%$ to $33.0 \%)$ at 10 years and 15 years (29.4\%, $24.2 \%$ to $35.2 \%)$.

Fewer than one in five survivors were active (as measured by the FAI) in their daily lives at both 10 and 15 years, and psychological impairments were identified in approximately one-third of survivors, with $39.1 \%$ (30.9\% to $47.9 \%$ ) screening positively for depression, $34.9 \%(27.0 \%$ to $43.8 \%)$ for anxiety and $30.0 \%$ (19.5\% to $43.1 \%)$ for cognitive impairment at 15 years. Stroke survivors reported poorer physical than mental HRQOL, with 15-year mean SF-12 physical and mental component summary scores of 35.3 and 44.1 , respectively.

The trends in the prevalence of functional, cognitive and psychological outcomes of stroke survivors alive at 10 years in the following 5 years are summarised in figure 2. In this group, the prevalence of functional, cognitive and psychological impairments was relatively stable up to 15 years. On average, between 10 and 15 years after stroke, $25 \%$ of survivors were moderatelyseverely disabled, $21 \%$ were inactive, $22 \%$ had cognitive impairments, 32\% were anxious and 38\% depressed.

Outcomes after 10 years were associated with the level of disability survivors had 10 years after stroke (figure 3 ).

When looking at 10-year survivors, who were independent in activities of daily living at 10 years after stroke, 42.3\% (27.1\% to $57.5 \%$ ) remained so at 15 years. In those with a mild disability at 10 years, over half $(53.1 \%, 35.1 \%$ to $71.2 \%)$ were either independent or had mild disability by 15 years. Survival outcomes in patients with moderate-severe disability at 10 years were poor, with a 5-year mortality rate of death of $67.2 \%$ $(55.5 \%$ to $76.0 \%)$. Outcomes stratified by disability level at 3 months after stroke were also estimated and results provided in online supplementary figure S2.

The health status of patients who had survived to 15 years are summarised in figure 4 . The prevalence of moderate to severe disability increased over time and 1 in 10 patients had lived with moderate-severe disability since the stroke. A gradual increase in the prevalence of inactivity, depression and cognitive impairment was also observed, while the prevalence of anxiety remained stable at around $31 \%$.

\section{DISCUSSION}

In this population-based cohort of people with first-ever stroke, we found that one in five lived to at least 15 years after stroke

Table 1 Characteristics of SLSR participants recruited from 1995 to 2003

\begin{tabular}{|c|c|c|c|}
\hline & Whole cohort & 10 years & 15 years \\
\hline Number alive and eligible for follow-up & 2626 & 723 & 262 \\
\hline Completed follow-up, $n(\%)$ & & 409 (56.7) & $133(50.8)$ \\
\hline $\begin{array}{l}\text { Number of deaths } \\
\text { Cumulative survival } \%(95 \% \mathrm{Cl})\end{array}$ & & $\begin{array}{l}1903 \\
28.9 \%(27.0 \% \text { to } 30.1 \%)\end{array}$ & $\begin{array}{l}2066 \\
20.6 \%(19.0 \% \text { to } 22.4 \%)\end{array}$ \\
\hline $\begin{array}{l}\text { Number of recurrences } \\
\text { Cumulative survival free of recurrence, } \%(95 \% \mathrm{Cl})\end{array}$ & & $\begin{array}{l}318 \\
65.3 \%(61.4 \% \text { to } 68.9 \%)\end{array}$ & $\begin{array}{l}333 \\
54.4 \%(47.4 \% \text { to } 60.9 \%)\end{array}$ \\
\hline Age at stroke onset, median (IQR) & $73(62.8-81.2)$ & $62.1(52.0-69.7)$ & $58.1(48.1-66.4)$ \\
\hline \multicolumn{4}{|l|}{ Sex, n $(\%(95 \% \mathrm{Cl}))$} \\
\hline Male & $1299(49.5(47.6$ to 51.4$))$ & $425(58.8$ (55.1 to 62.3$))$ & $159(60.7(54.6$ to 66.5$))$ \\
\hline Female & $1327(50.5$ (48.6 to 52.4$))$ & $298(41.2(37.7$ to 44.9$))$ & $103(39.3(33.5$ to 45.4$))$ \\
\hline \multicolumn{4}{|l|}{ Ethnicity, n (\% (95\% Cl)) } \\
\hline White & $1956(74.5(72.8$ to 76.1$))$ & $438(60.6(57.0$ to 64.1$))$ & $163(62.2(56.1$ to 67.9$))$ \\
\hline Black & $473(18.0$ (16.6 to 19.5$))$ & $214(29.6(26.4$ to 33.0$))$ & $77(29.4(24.2$ to 35.2$))$ \\
\hline Other & $197(7.5(6.6$ to 8.6$))$ & $71(9.8(7.9$ to 12.2$))$ & $22(8.4(5.6$ to 12.5$))$ \\
\hline \multicolumn{4}{|l|}{ Stroke subtype, n (\% (95\% Cl)) } \\
\hline Ischaemic & $1904(72.5(70.8$ to 74.2$))$ & 541 (74.8 (71.5 to 77.9$))$ & $189(72 \cdot 1(66 \cdot 4$ to $77 \cdot 3))$ \\
\hline $\mathrm{PICH}$ & $359(13.7(12.4$ to 15.0$))$ & 87 (12.0 (9.9 to 14.6$)$ & $34(13.0(9.4$ to 17.6$))$ \\
\hline $\mathrm{SAH}$ & $157(6.0(5.1$ to 7.0$))$ & $72(10.0(8.0$ to 12.4$))$ & $32(12.2$ (8.7 to 16.8$))$ \\
\hline Unclassified/unknown & $206(7.8(6.9$ to 8.9$))$ & $23(3.2(2.1$ to 47$))$ & $7(2.7(1.3$ to 5.5$))$ \\
\hline
\end{tabular}


Table 2 Prevalence of outcomes at 10 and 15 years after stroke

10-year survivors, $\mathbf{n}(\%(95 \% \mathrm{Cl}))$

15-year survivors, $\mathrm{n}(\%(95 \% \mathrm{CI}))$ )

\section{Living conditions}

Private household alone/with others

$372(85.1$ (81.5 to 88.2))

Nursing home

Institution (long-term hospital care)

26 (6.0 (4.1 to 8.6))

$32(7.3(5.2$ to 10.2$))$

Other/unknown

7 (1.6 (0.7 to 3.3$))$

25 (86.8 (80.1 to 91.5$))$

arthel index

Independent

135 (33.0 (28.6 to 37.3))

Mild disability

165 (40.3 (35.7 to 45.2$))$

50 (12.2 (9.4 to 15.8$))$

59 (14.4 (11.3 to 18.2))

0

Moderate disability

66 (17.0 (13.6 to 21.1))

18 (12.5 (8.0 to 19.1$))$

Severe disability

$66(17.0(13.6$ to 21.1$))$
$130(33.5(29.0$ to 38.4$)$

1 (0.69 (0.7 to 3.3$))$

renchay Activities Index

Active

192 (49.5 (44.5 to 54.5 )

49 (36.8 (29.0 to 45.4$))$

45 (33.8 (26.2 to 42.4))

19 (14.3 (9.2 to 21.4$))$

Moderately active

$244(64.4(59.4$ to 69.1$))$

20 (15.0 (9.9 to 22.3))

Inactive

135 (35.6 (30.9 to 40.6$))$

23 (18.3 (12.4 to 26.1))

HAD's - depression

Not depressed

48 (38.1 (29.9 to 47.0$))$

55 (43.7 (35.2 to 52.5$))$

Depressed

260 (68.6 (63.7 to 73.1$))$

78 (60.9 (52.1 to 69.1))

$H A D^{\prime} s$ - anxiety

Not anxious

119 (31.4 (26.9 to 36.3))

50 (39.1 (30.9 to 47.9))

Anxious

172 (72.0 (65.9 to 77.3$))$

82 (65.1 (56.2 to 73.0))

Cognitive state

Cognitively intact

67 (28.0 (22.7 to 34.1$))$

42 (70.0 (56.9 to 80.5))

Cognitively impaired

34.7 (33.6 to 35.8$)$

18 (30.0 (19.5 to 43.1))

uality of life, mean $(95 \% \mathrm{Cl})$

SF-12 physical summary score

SF-12 mental summary score

46.3 (45.2 to 47.4$)$

35.3 (33.4 to 37.2 )

44.1 (42.2 to 46.2 )

HADS, Hospital Anxiety and Depression Scale; SF-12, Short Form-12.

and poor outcomes were common in these long-term survivors, at a prevalence similar to that observed in the short term after stroke. Within each of the domains assessed, around one-third had poor outcome at any given time. If these figures are representative of stroke outcomes in other countries, this implies that $\sim 3.4$ million of those having stroke each year globally will be alive 15 or more years after stroke. It is not certain whether longterm survival and outcomes will be similar in other settings, particularly countries with less advanced healthcare systems, but nonetheless these findings help to quantify what projections from the Global Burden of Disease study ${ }^{1}$ will mean in terms of the future burden of stroke on individuals, health systems and society as more patients survive stroke to experience stroke as a longterm condition. Finally, they also highlight the need for more research and health service improvement focused on preventing and managing the long-term consequences of stroke.

Previous studies of stroke outcomes have been limited to relatively short follow-up durations, with only a small number of studies reporting outcomes more than 5 years after stroke. ${ }^{4-15}$ The largest previous study of long-term stroke outcomes was a survey of patients with stroke admitted to hospital with stroke in Finland in the 1970s, of whom 19\% were alive 14 years after stroke, a similar proportion to the current study. ${ }^{13}$ Of these, $\sim 30 \%$ self-reported severe disability and 40\% minor disability, and psychological symptoms were common, with over half of the responders reporting that they had low mood or depression.

A similar prevalence of disability was observed at 10 years in a cohort of 45 stroke survivors in a population-based register from Australia, in whom the cumulative risk of disability was $44 \%$ at 10 years. $^{12}$ Survival and quality of life were reported at 21 years in 50 patients recruited in a population-based register in Auckland, New Zealand. ${ }^{10}$ Quality of life was considered acceptable in the majority of respondents and 19\% required help with ADL-of these, most (8 of 9) considered functional impairments to be attributable to the stroke. The longest follow-up of stroke outcomes reported until now was carried out as part of the Framingham cohort study, which described outcomes in 10 people who had lived at least 20 years after stroke. ${ }^{11}$ Outcomes in this small group were generally good; most (8 of 10) were independent in ADL and able to drive a car and only one (11\%) reported depression or cognitive impairment. However, all of these long-term cohort studies are limited by small numbers and are now quite historic, and may not reflect more contemporary stroke outcomes or treatments (such as stroke unit based care or thrombolysis).

The SLSR has also previously reported outcomes up to 10 years after stroke. ${ }^{9}$ This found that between $20 \%$ and $30 \%$ of patients with stroke had a range of poor outcomes up to 10 years after stroke, providing evidence that stroke is associated with long-term health outcomes. We have extended this previous study in several ways: first, we have provided a further 5 years of long-term follow-up demonstrating a similar pattern of health outcomes and providing uniquely detailed data on outcomes in very long-term stroke survivors. Second, we have used analytic approaches and data visualisations that focus on cumulative outcomes, better aiding in providing patient level prognostic estimates and in describing outcomes in patients with incident stroke. For example, the estimates from this study could be used in sharing information about prognosis with patients with stroke and their families, as well as in planning healthcare services for long-term stroke survivors. Equivalent long-term outcomes data for other long-term conditions are 
A

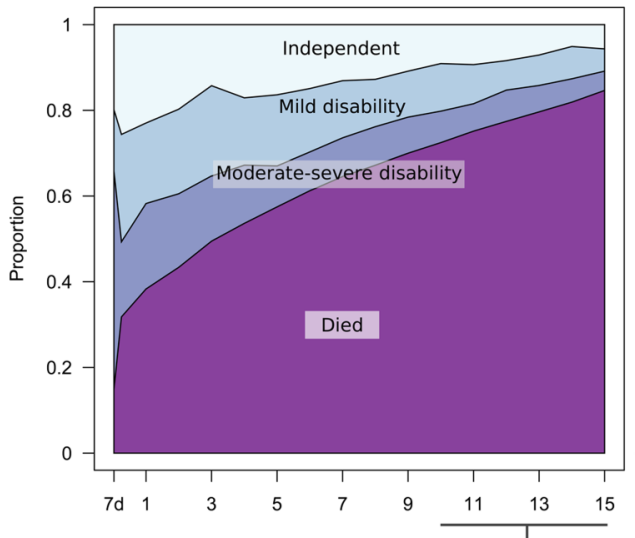

B 10 years-15 years
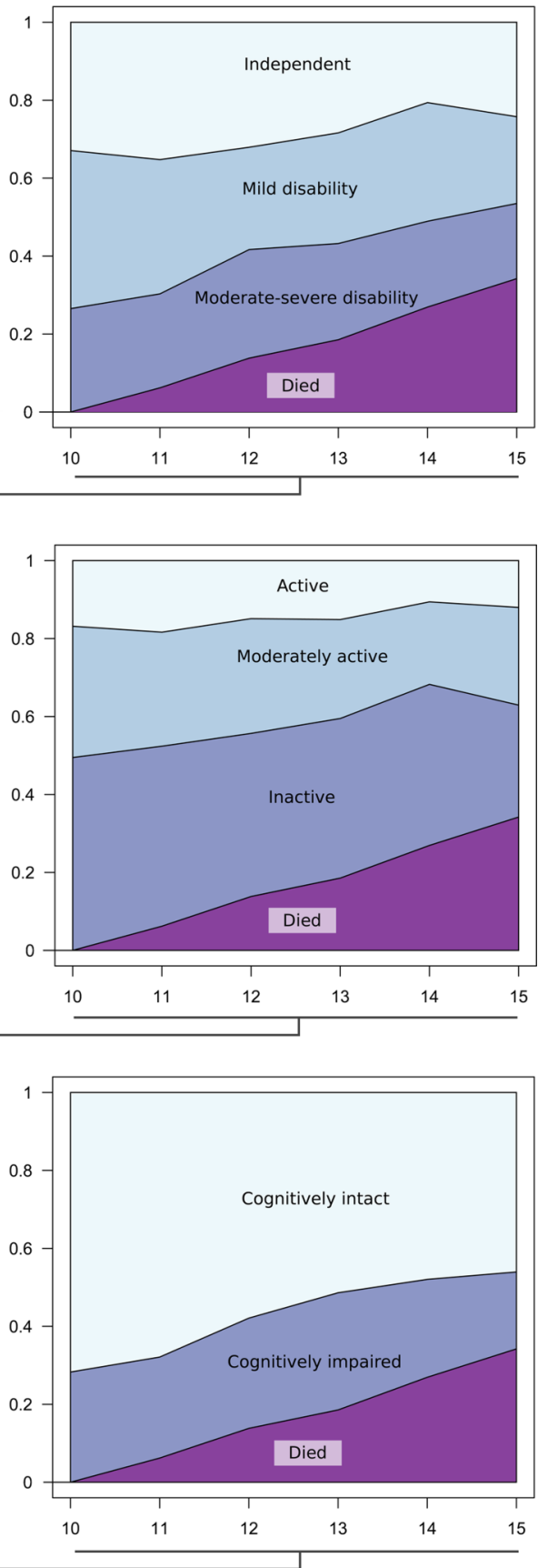

Figure 2 Functional, cognitive and psychological outcomes between 10 and 15 years after stroke. HADs, Hospital Anxiety and Depression scale.

generally lacking and largely limited to survival only; these data therefore provide a relatively unique insight into the long-term outlook across a broad array of health outcomes for patients with cardiovascular long-term conditions.

One of the challenges in interpreting these data is in ascertaining to what extent the observed outcomes are due to stroke itself versus the effect of accumulating multimorbidity or advancing age. Multimorbidity in patients with stroke ${ }^{30}$ and in general populations ${ }^{31}$ is common and the health outcomes of the patients in this study will be the result of both accumulating multimorbidity and of the stroke itself. Nonetheless, these data show that although there was some accumulation of poor health outcomes in survivors over time, many of the patients with physical disability at 15 years had lived with disability since their stroke; stroke can directly impact long-term health and is not just a risk factor or marker for subsequent health decline. The data from these long-term survivors are also evidence that some people (generally younger) with stroke may survive for many years after stroke despite having severe disability and that poor health outcomes do not decline in prevalence with time. This highlights the need to focus research and health service improvement not just on acute stroke care but also on the problems faced by long-term stroke survivors.

The strengths of this study include the use of a populationbased register specifically designed to identify all strokes occurring in the defined cohort, reducing the risk of selection bias. The study also used longitudinal follow-up at multiple time points, enabling the long-term trajectory of stroke outcomes to 

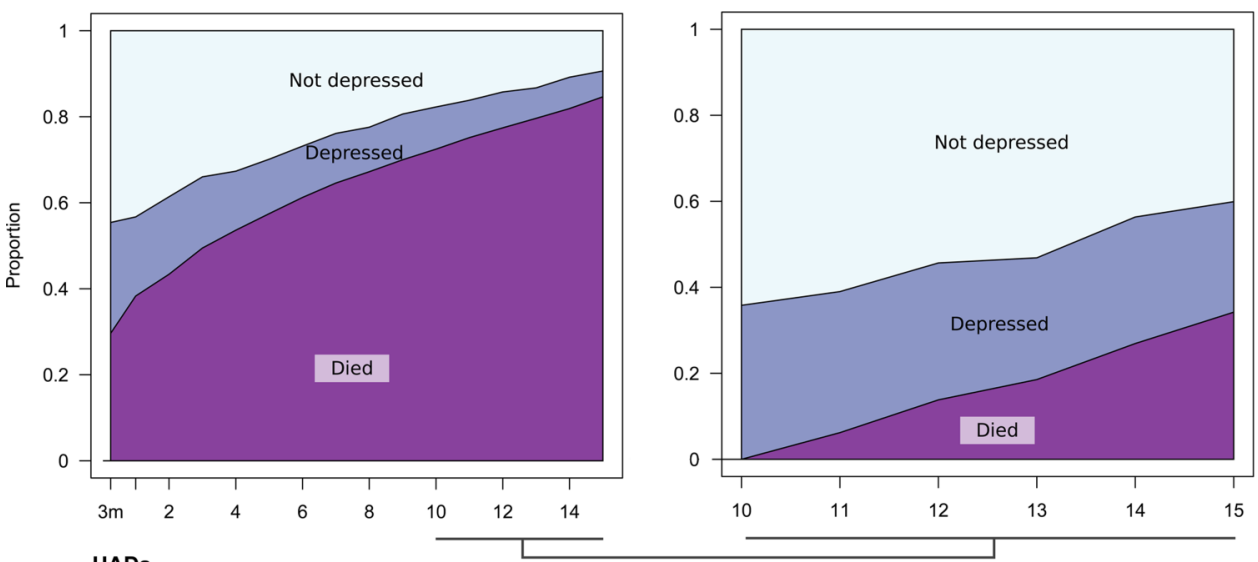

HADs -
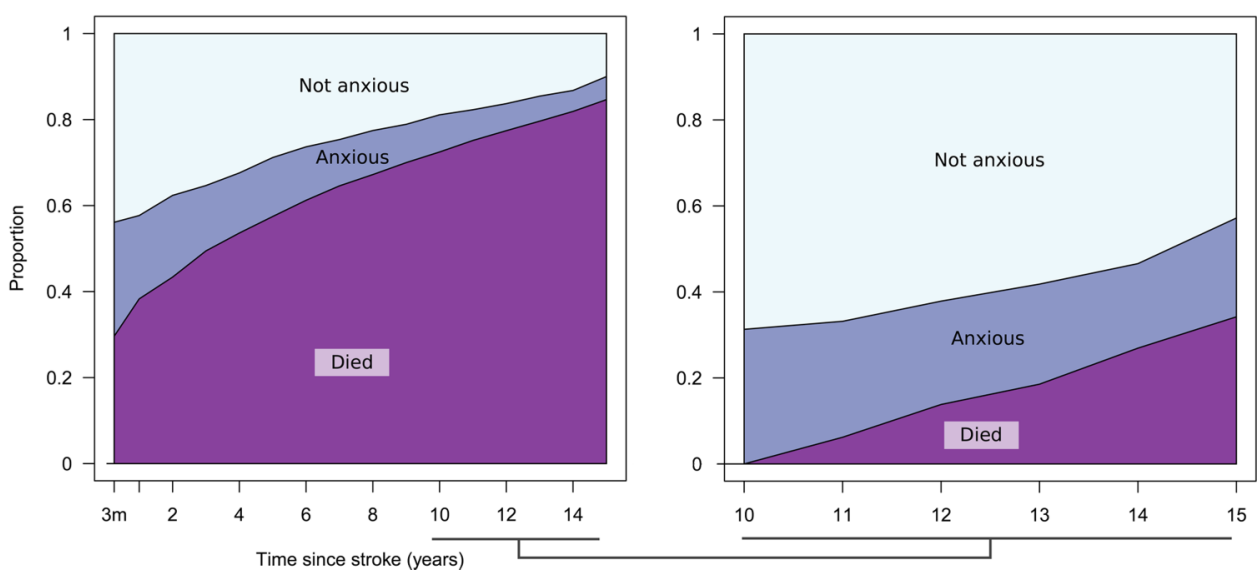

Figure 2 Continued

be described and reducing the impact of missing data. Data collection was standardised through the use of validated instruments and through data collection by trained fieldworkers, helping to maintain accuracy and consistency.

The main limitation of the study is missing data for patients who were lost to follow-up or left the area-maintaining contact in very long-term cohort studies is challenging and multiple methods were used for tracing and to contact patients who moved out of the area. While this study reports on data from one of the largest cohorts of stroke survivors until now, owing to high mortality rates up to 10 years after stroke, only a quarter of the initial cohort were eligible to be included. Incomplete follow-up data reduced the number further and have the potential to introduce bias and reduce the precision of estimates. However, we found that the only major variable associated with missing data was age 10 years after stroke. We have carried out simulation studies which found that the results are robust to the observed pattern of missing data and not meaningfully improved by multiple imputation (Crichton- $\mathrm{PhD}$ Thesis currently in submission). The setting of the study in an inner city area of London also means that the source population of the study area is unlikely to be representative of the UK population as a whole; in particular, the proportion of the population who are from non-white ethnic groups is around 30\% point higher than that for the UK as a whole. We observed a greater proportion of long-term survivors to be of black ethnicity than among the whole cohort. It has previously been shown that in the SLSR the average age at onset among the black population is 10 years lower in those of white ethnicity ${ }^{18}$ and so the proportion of stroke survivors surviving beyond 10 years may be lower in the general population. This study also lacked a control group of patients without stroke, which would have strengthened the study and enabled comparisons of health status with people without stroke. This is a fundamental aspect of the design of the cohort study that cannot be changed retrospectively, but we are exploring the use of data linkage that will enable us in the future to sample a control group from the local population using electronic health records. Other limitations are that the assessment of mental health and cognitive outcomes made use of screening instruments rather than full neuropsychological or clinical assessments and that the data do not include measurements of some outcomes that stroke survivors identify as being important, such as fatigue. ${ }^{32}$ Other factors that were collected, such as recurrent stroke, are likely to impact on longterm outcomes. However, the aim of this study was to describe outcomes for a population, and describing the impact of factors on outcomes was outside the scope of this work. Similarly, the data set did not include some potentially relevant variables that contribute to health status, such as marital status and level of family support. In practice, more comprehensive assessments would have been logistically difficult to administer given the size of the cohort and long duration of the SLSR, and the data remain one of the most detailed descriptions of the long-term outcomes of a cardiovascular long-term condition ever collected.

\section{CONCLUSION}

This study provides some of the most detailed, year-on-year estimates of the very long-term outcomes of stroke, describing not just survival but also outcomes across a broad range of physical, quality of life and mental health domains. As well as being useful prognostically, these data help to quantify the impact on populations of the epidemiological shift of stroke towards a 

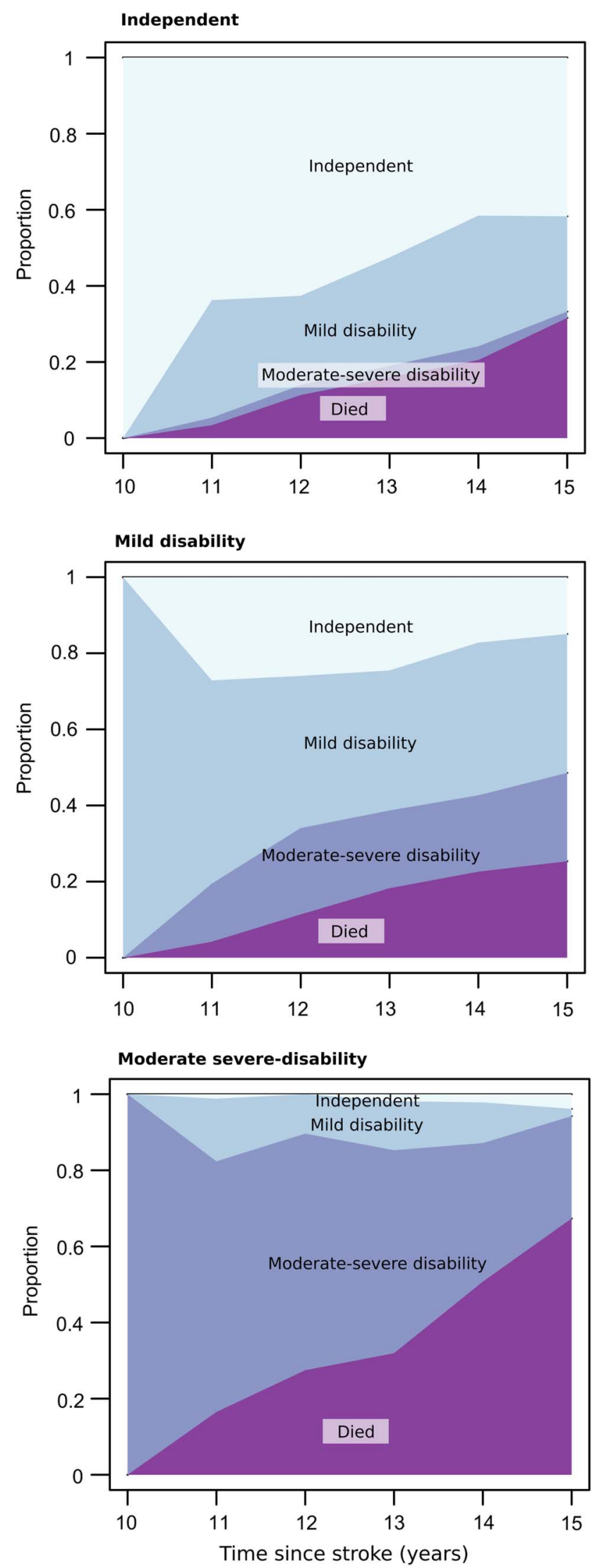

Figure 3 Barthel Index between 10 and 15 years after stroke stratified by functional status at 10 years after stroke.

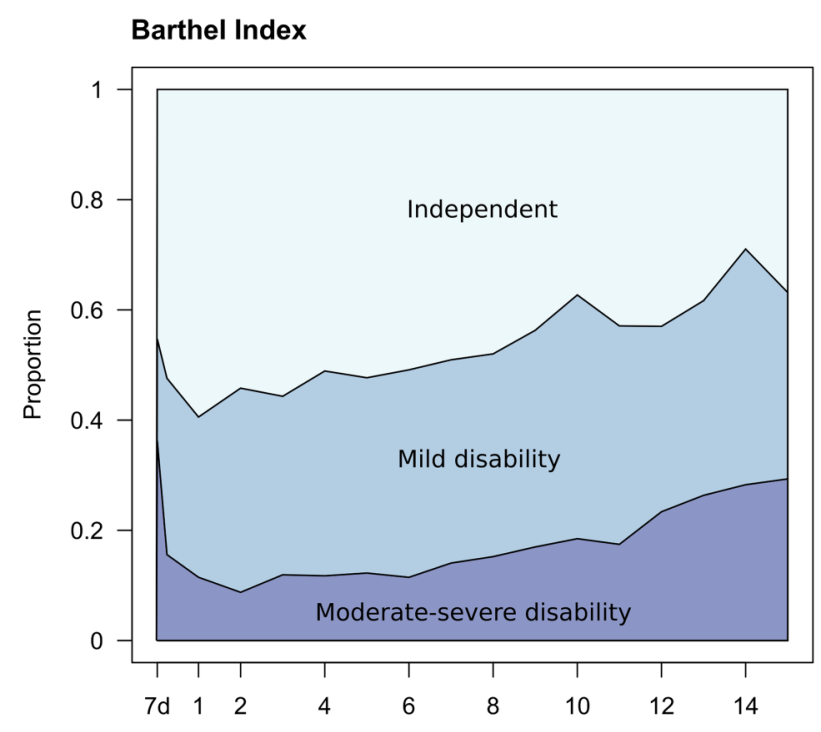

\section{Frenchay Activities Index}

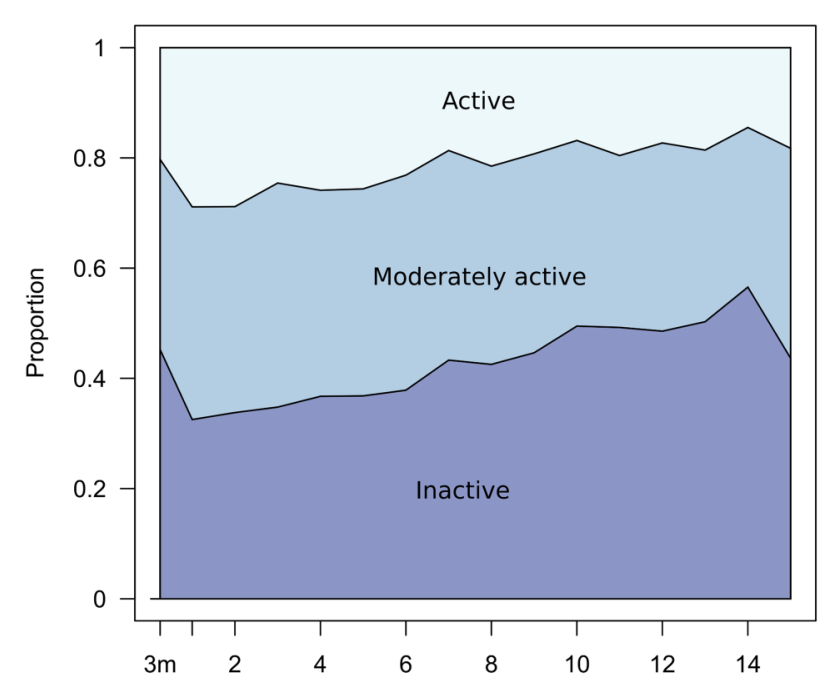

Cognitive status

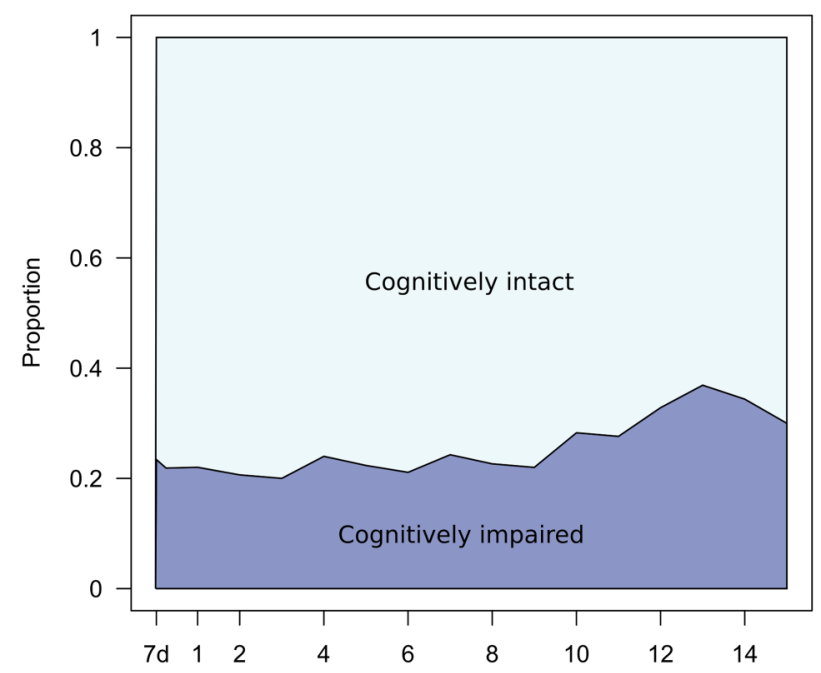

Figure 4 Functional, cognitive and psychological outcomes of 15-year survivors. HADs, Hospital Anxiety and Depression scale. 
HADs- Depression

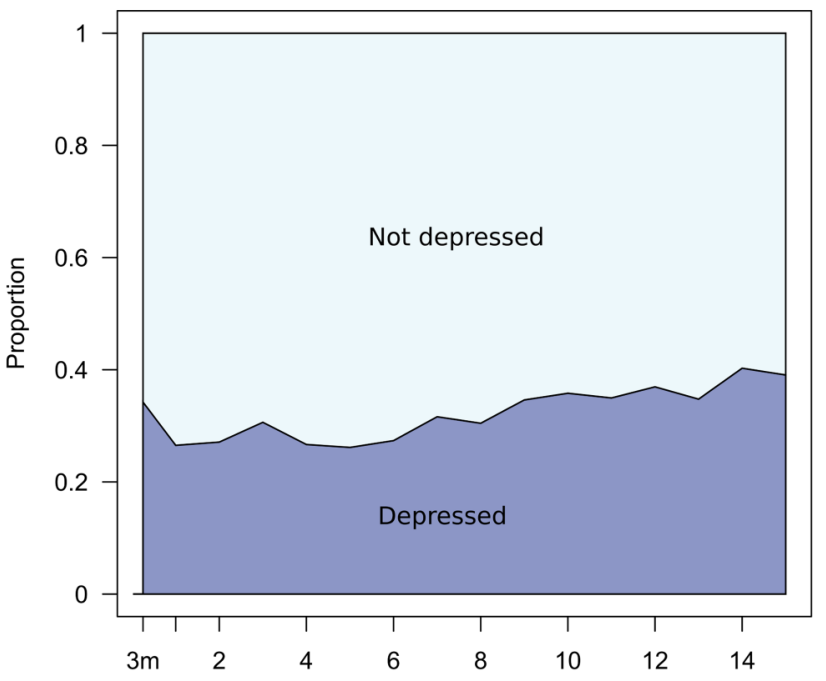

HADs - Anxiety

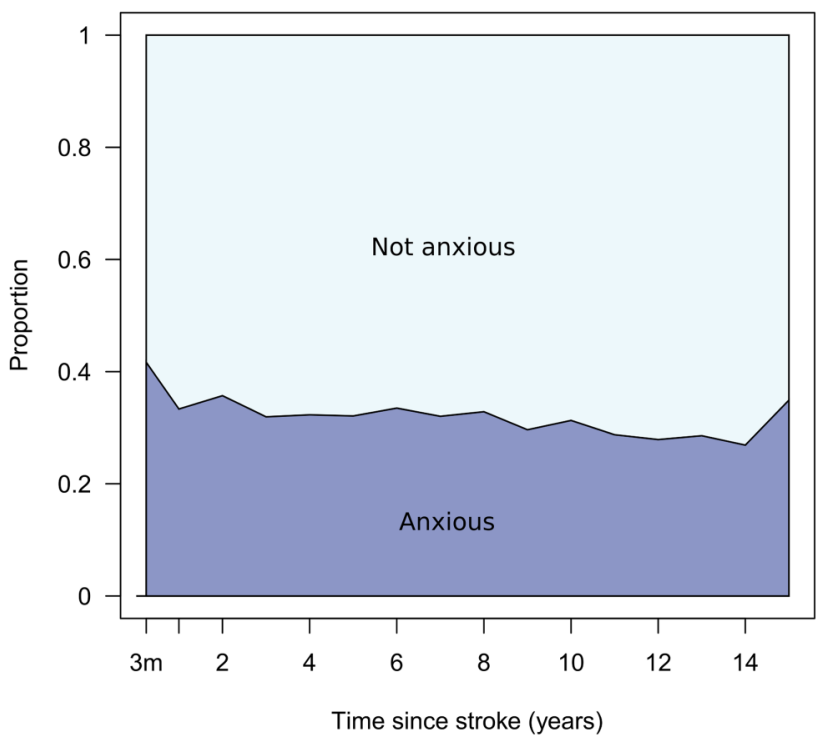

Figure 4 Continued

long-term condition and highlight the need to focus not just on reducing stroke mortality, but on preventing and managing the long-term consequences of stroke.

Twitter Follow Benjamin Bray at @benthebray

Contributors CDAW and AGR secured the funding. BDB, SLC, CMK, AGR and CDAW designed the study. BDB and SLC conducted the analysis. BDB, SLC, CMK, AGR and CDAW co-wrote the paper.

Funding The research was funded/supported by the National Institute for Health Research (NIHR) Biomedical Research Centre based at Guy's and St Thomas' NHS Foundation Trust and King's College London, and the NIHR Collaboration for Leadership in Applied Health Research and Care South London at King's College Hospital NHS Foundation Trust. The views expressed are those of the authors and not necessarily those of the NHS, the NIHR or the Department of Health.

Competing interests None declared.

Ethics approval Guy's and St Thomas' NHS Foundation Trust.

Provenance and peer review Not commissioned; externally peer reviewed.

\section{REFERENCES}

1 Feigin $\mathrm{V}$, Forouzanfar $\mathrm{M}$, Krishnamurthi R, et al. Global Burden of Diseases, Injuries, and Risk Factors Study 2010 (GBD 2010) and the GBD Stroke Experts Group.
Global and regional burden of stroke during 1990-2010: findings from the Global Burden of Disease Study 2010. Lancet 2014;383:245-54.

2 Lackland DT, Roccella EJ, Deutsch AF, et al. Factors influencing the decline in stroke mortality: a statement from the American Heart Association/American Stroke Association. Stroke 2014;45:315-53.

3 Feigin VL, Lawes CM, Bennett DA, et al. Worldwide stroke incidence and early case fatality reported in 56 population-based studies: a systematic review. Lancet Neurol 2009:8:355-69.

4 Pendlebury ST, Rothwell PM. Prevalence, incidence, and factors associated with pre-stroke and post-stroke dementia: a systematic review and meta-analysis. Lancet Neurol 2009:8:1006-18.

5 Hackett ML, Köhler S, O'Brien JT, et al. Neuropsychiatric outcomes of stroke. Lancet Neurol 2014:13:525-34.

6 Tobias M, Cheung J, Carter K, et al. Stroke surveillance: population-based estimates and projections for New Zealand. Aust N Z J Public Health 2007:31:520-5.

7 Jönsson $A C$, Delavaran $\mathrm{H}$, Iwarsson $\mathrm{S}$, et al. Functional status and patient-reported outcome 10 Years after stroke the Lund Stroke Register. Stroke 2014;45:1784-90.

8 Luengo-Fernandez R, Paul NL, Gray AM, et al. Population-based study of disability and institutionalization after transient ischemic attack and stroke: 10-year results of the Oxford Vascular Study. Stroke 2013:44:2854-61.

9 Wolfe CD, Crichton SL, Heuschmann PU, et al. Estimates of outcomes up to ten years after stroke: analysis from the prospective South London Stroke Register. PLoS Med 2011;8:e1001033.

10 Anderson CS, Carter KN, Brownlee WJ, et al. Very long-term outcome after stroke in Auckland, New Zealand. Stroke 2004;35:1920-4.

11 Gresham GE, Kelly-Hayes M, Wolf PA, et al. Survival and functional status 20 or more years after first stroke: the Framingham Study. Stroke 1998;29:793-7.

12 Hardie K, Hankey GJ, Jamrozik K, et al. Ten-year risk of first recurrent stroke and disability after first-ever stroke in the Perth Community Stroke Study. Stroke 2004;35:731-5.

13 Tuomilehto J, Nuottimäki T, Salmi K, et al. Psychosocial and health status in stroke survivors after 14 years. Stroke 1995;26:971-5.

14 van Wijk I, Gorter JW, Lindeman E, et al. Mental status and health-related quality of life in an elderly population 15 years after limited cerebral ischaemia. J Neurol 2007;254:1018-25.

15 van Wijk I, Lindeman E, Kappelle L, et al. Functional status and use of healthcare facilities in long-term survivors of transient ischaemic attack or minor ischaemic stroke. J Neurol Neurosurg Psychiatry 2006;77:1238-43.

16 Briffa T, Hickling $S$, Knuiman $M$, et al. Long term survival after evidence based treatment of acute myocardial infarction and revascularisation: follow-up of population based Perth MONICA cohort, 1984-2005. BMJ 2009;338:b36.

17 Vaccarino V, Berkman LF, Krumholz HM. Long-term outcome of myocardial infarction in women and men: a population perspective. Am J Epidemiol 2000;152:965-73.

18 Heuschmann PU, Grieve AP, Toschke AM, et al. Ethnic group disparities in 10-year trends in stroke incidence and vascular risk factors: the South London Stroke Register (SLSR). Stroke 2008;39:2204-10.

19 Tilling $K$, Sterne JA, Wolfe CD. Estimation of the incidence of stroke using a capture-recapture model including covariates. Int J Epidemiol 2001;30:1351-9.

20 Hillen T, Coshall C, Tilling K, et al. Cause of stroke recurrence is multifactorial patterns, risk factors, and outcomes of stroke recurrence in the south London stroke register. Stroke 2003;34:1457-63.

21 Wade D, Collin C. The Barthel ADL Index: a standard measure of physical disability? Int Disabil Stud 1988;10:64-7.

22 Ware JE, Kosinski M, Keller SD. SF-12: How to score the SF-12 physical and mental health summary scales. Health Institute, New England Medical Center, 1995.

23 Hodkinson HM. Evaluation of a mental test score for assessment of mental impairment in the elderly. Age Ageing 1972;1:233-8.

24 Zigmond AS, Snaith RP. The hospital anxiety and depression scale. Acta Psychiatr Scand 1983:67:361-70.

25 Wolfe CD, Taub NA, Woodrow EJ, et al. Assessment of scales of disability and handicap for stroke patients. Stroke 1991;22:1242-4.

26 Anderson CS, Jamrozik KD, Broadhurst RJ, et al. Predicting survival for 1 year among different subtypes of stroke. Results from the Perth Community Stroke Study. Stroke 1994;25:1935-44.

27 Jitapunkul S, Pillay I, Ebrahim S. The abbreviated mental test: its use and validity. Age Ageing 1991;20:332-6.

28 Bjelland I, Dahl AA, Haug TT, et al. The validity of the Hospital Anxiety and Depression Scale. An updated literature review. J Psychosom Res 2002:52:69-77.

29 Pickard AS, Johnson JA, Penn A, et al. Replicability of SF-36 summary scores by the SF-12 in stroke patients. Stroke 1999;30:1213-17.

30 Gallacher KI, Batty GD, McLean G, et al. Stroke, multimorbidity and polypharmacy in a nationally representative sample of 1,424,378 patients in Scotland: implications for treatment burden. BMC Med 2014;12:151.

31 Barnett K, Mercer SW, Norbury M, et al. Epidemiology of multimorbidity and implications for healthcare, research, and medical education: a cross-sectional study. Lancet 2012;380:37-43.

32 McKevitt C, Fudge N, Redfern J, et al. Self-reported long-term needs after stroke. Stroke 2011;42:1398-403. 